\title{
Experimental Approach to Search for Free Neutron-Antineutron Oscillations Based on Coherent Neutron and Antineutron Mirror Reflection
}

\author{
V. V. Nesvizhevsky, ${ }^{1, *}$ V. Gudkov, ${ }^{2}$ K. V. Protasov, ${ }^{3}$ W. M. Snow, ${ }^{4}$ and A. Yu. Voronin ${ }^{5}$ \\ ${ }^{1}$ Institut Max von Laue-Paul Langevin, 71 avenue des Martyrs, Grenoble, France 38042 \\ ${ }^{2}$ Department of Physics and Astronomy, University of South Carolina, Columbia, South Carolina 29208, USA \\ ${ }^{3}$ Laboratoire de Physique Subatomique et de Cosmologie, UGA-CNRS/IN2P3, Grenoble, France 38026 \\ ${ }^{4}$ Department of Physics, Indiana University, 727 E. Third St., Bloomington, Indiana 47405, USA \\ ${ }^{5}$ P.N. Lebedev Physical Institute, 53 Leninsky prospect, Moscow, Russia 119991
}

(Received 1 October 2018; revised manuscript received 6 February 2019; published 7 June 2019)

\begin{abstract}
An observation of neutron-antineutron oscillations $(n-\bar{n})$, which violate both $B$ and $B-L$ conservation, would constitute a scientific discovery of fundamental importance to physics and cosmology. A stringent upper bound on its transition rate would make an important contribution to our understanding of the baryon asymmetry of the Universe by eliminating the postsphaleron baryogenesis scenario in the light quark sector. We show that one can design an experiment using slow neutrons that in principle can reach the required sensitivity of $\tau_{n-\bar{n}} \sim 10^{10} \mathrm{~s}$ in the oscillation time, an improvement of $\sim 10^{4}$ in the oscillation probability relative to the existing limit for free neutrons. The improved statistical accuracy needed to reach this sensitivity can be achieved by allowing both the neutron and antineutron components of the developing superposition state to coherently reflect from mirrors. We present a quantitative analysis of this scenario and show that, for sufficiently small transverse momenta of $n / \bar{n}$ and for certain choices of nuclei for the $n / \bar{n}$ guide material, the relative phase shift of the $n$ and $\bar{n}$ components upon reflection and the $\bar{n}$ annihilation rate can be small enough to maintain sufficient coherence to benefit from the greater phase space acceptance the mirror provides.
\end{abstract}

DOI: 10.1103/PhysRevLett.122.221802

The possible existence of neutron-antineutron $(n-\bar{n})$ oscillations is of fundamental interest for particle physics and cosmology. $n-\bar{n}$ oscillations would violate baryon number $(\Delta B=2)$ and have many other implications for new physics [1-39]. Sensitive searches for $\Delta B=2$ processes, especially with $\Delta(B-L)=2$ such as $n-\bar{n}$, have started to attract more scientific attention. Cosmological arguments which use the Sakharov criteria [2] to generate the baryon asymmetry of the Universe starting from a $B=0$ condition require $B$ violation. A baryon asymmetry generated above the electroweak scale and conserving $B-L$, such as $\Delta B=1$ and $\Delta L=1$ proton decay $p \rightarrow \pi_{0} e^{+}$, could be erased at the electroweak phase transition by sphalerons. Several theoretical models possess $\Delta B=2$ processes leading to $n-\bar{n}$ without giving $p$ decay [23-25,28,29,33,34,37].

An especially interesting class of models collectively referred to as postsphaleron baryogenesis (PSB) [34] can generate the baryon asymmetry below the electroweak

Published by the American Physical Society under the terms of the Creative Commons Attribution 4.0 International license. Further distribution of this work must maintain attribution to the author(s) and the published article's title, journal citation, and DOI. Funded by SCOAP ${ }^{3}$. scale. Experiments proposed here can rule out PSB models operating in the light quark sector in combination with constraints on other consequences of the model from the LHC. By falsifying this model, an understanding of electroweak sphaleron physics may be required to explain the baryon asymmetry within the Sakharov paradigm.

New analyses of existing data to constrain $\Delta B=2$ processes have appeared from SuperK [40] and SNO [41]. The possibility of more sensitive $B$ violation searches in future underground detectors such as HyperK [42] and Dune [43] has lead to new work on $\bar{n} A$ dynamics [44]. An observation of $n-\bar{n}$ would put stringent limits on $C P T$ violation in the nucleon sector [45] within the effective field theory for $C P T$ or Lorentz violation known as the standard model extension [46]. $n-\bar{n}$ would constrain long-range gauge fields coupled to $B-L$, and improve on present constraints from tests of the equivalence principle over a broad range of couplings and ranges [47,48]. $n-\bar{n}$ oscillations in combination with other data can imply that the neutrino is a Majorana particle $[45,49]$. Recent theoretical studies [50-54] have clarified the subtleties involved in properly understanding the discrete symmetry transformations of a composite strongly interacting bound system like the $n$. A new $\Delta B=2$ process, $n-\bar{n}$ conversion, has been identified and described [52,55], and the possibility of $n$-mirror $n$ oscillations [56,57], indicated as a 
possibility in experiments [58-61] using ultracold neutrons (UCNs) $[62,63]$ is the subject of active studies [64]. Recent studies have investigated in greater depth the limits of the so-called quasifree condition for the evolution of the $n / \bar{n}$ amplitudes in external magnetic fields [65-67]. These developments in theory and experiment show that the approach described here for improving the sensitivity for $n-\bar{n}$ oscillations is of general interest to the physics community.

Experimental searches for $\Delta B=2$ processes involving $n$ have so far been conducted in two ways. Free $n$ oscillation searches have been designed so that $n$ avoids interactions with matter and external fields. Despite the fact that $\Delta E \gg \varepsilon$ ( $\varepsilon$ is the off-diagonal mixing term in the effective Hamiltonian for the $n / \bar{n}$ two-state system), the oscillation rate is not greatly suppressed if the observation time $t$ is short compared to $\Delta E / \hbar$ ( $\hbar$ is the reduced Planck constant). In this quasifree regime, the relative phase shift between the $n$ and $\bar{n}$ states, $e^{-\Delta E t / \hbar}$, is small enough that the oscillation probability still grows quadratically with $t$. The other approach searches for $n-\bar{n}$ oscillations of the $n$ bound in nuclei, where the rate is suppressed by a very large $\Delta E$ [9,68-73]. The very large number of $n$ in large volume of low-background underground detectors makes this the most sensitive search mode at present. However, the interpretation of the results depends somewhat on models of $\bar{n}$ annihilation in nuclei and the branching ratios for the different reaction products from the $\bar{n}$ annihilation. Also, this process is not equivalent to free $n$ oscillations due to additional $\Delta B=2$ processes which can happen inside a nucleus but not for a free $n$. Therefore, the bounds from these two approaches are complementary.

We propose and analyze a new version of $n-\bar{n}$ experiment: an almost free $n$ oscillation search in which we allow slow $n / \bar{n}$ (with energies of $<10^{-2} \mathrm{eV}$ ) to reflect from $n / \bar{n}$ optical mirrors. Although reflection of $n-\bar{n}$ was considered already in 1980 for UCNs $[8,12,74,75]$ for experiments constraining $\tau_{n-\bar{n}}$, we extend this approach to higher energies (slow $n$ that can be confined in neutron guides), point out conditions for suppressing the phase difference for $n$ and $\bar{n}$, quantify the low transverse momenta of $n / \bar{n}$ required, and make new choices for the nuclei composing the guide material. We show that, over a broad fraction of phase space acceptance of a $n / \bar{n}$ guide, the probability of coherent reflection of $n / \bar{n}$ from the walls can be high, the relative phase shift can be small, and the theoretical uncertainties in the calculation of the experimental sensitivity can be small. We show that such an experimental mode can relax some of the constraints on free $n$ oscillation searches and in principle allow us to achieve a much higher sensitivity. This approach can preserve both the very low antineutron detector backgrounds that have been achieved in free $n$ oscillation searches and the ability to confirm a nonzero signal by applying a small external magnetic field on the beam to split the $n$ and $\bar{n}$ states by $\Delta E=2 \mu B$ enough to suppress the oscillation probability. However, it does not require the same level of detail in the understanding of the $\bar{n}$ dynamics and the subsequent annihilation products needed to interpret the underground detector $\bar{n}$ annihilation experiments.

For slow neutrons the $\bar{n}$ coherent scattering amplitude comes from a single $s$-wave scattering length whose real and imaginary parts can be calculated within a phenomenological model [76] reflecting a simple geometrical picture of $\bar{n} A$ annihilation. The strong $\bar{n}$ absorption on the nuclear surface means that the real part of the scattering amplitude is very close to the nuclear size plus the nuclear skin thickness, and the imaginary part of the scattering amplitude is approximately the same for all nuclei. As long as the mirror materials are neither ferromagnetic nor ferroelectric, neither the internal magnetic fields $\vec{B}$ nor the motional magnetic fields from $\vec{v} \times \vec{E}$ are large enough to violate the quasifree condition for the very short time while the neutron is inside matter during the reflection even if one takes into account dispersion corrections to the neutron optical potential [77-81], nor do these internal fields create phase noise in the amplitudes for coherent scattering [82-87]. Note that the $n-\bar{n}$ oscillations of interest happen in vacuum while the forward scattering only guarantees coherent reflection from the walls and does not induce the oscillations as in Ref. [55], thus allowing us to distinguish these two processes.

A general expression for the $n-\bar{n}$ oscillation probability $P_{n \rightarrow \bar{n}}$ [88] resembles the well-known equation for the neutral kaon oscillations. For practical observation times, $e^{-\Gamma_{\beta} t} \approx 1$ ( $\Gamma_{\beta}$ is the $n \beta$-decay width), $\omega t \ll 1(\omega$ is the oscillation frequency), $P_{n \rightarrow \bar{n}}(t) \approx \varepsilon^{2} e^{-\left(\Gamma_{\alpha} t / 2\right)} t^{2}$, where $\Gamma_{\alpha}$ is the $\bar{n}$ annihilation width. For the optimum observation time $t_{0}=4 / \Gamma_{\alpha}$, the corresponding $n-\bar{n}$ oscillation probability is

$$
P_{n \rightarrow \bar{n}} \approx 2.1\left(\frac{\varepsilon}{\Gamma_{\alpha}}\right)^{2}
$$

Neglecting annihilation, it reduces to the quasifree limit expression $P_{n \rightarrow \bar{n}} \approx\left(t / \tau_{n \rightarrow \bar{n}}\right)^{2}$, where $\tau_{n \rightarrow \bar{n}}=1 /|\varepsilon|$ (for natural units $\hbar=c=1)$ is the oscillation time. As a few annihilation events suffice for a positive signal, the figure of merit is $F \approx N t^{2}$ with the total number $N$ of $n$. In the limit of small widths, it reduces to

$$
P_{n \rightarrow \bar{n}}=\frac{2 \varepsilon^{2}}{\omega^{2}}[1-\cos (\omega t)] \approx \varepsilon^{2} t^{2}\left[1-\frac{1}{12}(\omega t)^{2}\right],
$$

and even a phase shift of $1 \mathrm{rad}$ would give only an $\sim 8 \%$ correction.

The best constraint on $\tau_{n \rightarrow \bar{n}}$ with free $n$ used an intense cold neutron beam at the Institute Laue-Langevin (ILL) [89] which built on earlier searches [90,91]. An ambitious project at a projected fundamental physics beam line at the 
European Spallation Source (ESS) [92] proposes an analogous scheme to increase the sensitivity by a factor $G \approx$ $10^{2}-10^{3}$ but requires a large solid angle neutron extraction from the source. We argue that the sensitivity can be improved if one allows the reflection of $n / \bar{n}$ from mirrors to increase the observation time $t$ and the counting statistics. Some literature on the subject gives the incorrect impression that the coherence of the $n / \bar{n}$ amplitude is always destroyed upon contact with matter. This is not true for a coherent neutron reflection from a surface. As long as the $\bar{n}$ is not "observed" (annihilated) and the phase difference between the $n$ and $\bar{n}$ components of the amplitude upon reflection is small, the quasifree condition is met and the sensitivity for the $\bar{n}$ component grows quadratically with time. We evaluate an experiment at the PF1B beam [93] at ILL as an example. Greater sensitivity could be achieved at other neutron sources or guides. A combined design including the extraction of neutrons through a large solid angle as in Ref. [92] could provide additional improvements. To estimate the sensitivity for different configurations, one can use standard neutron optical calculations with the formalism developed here.

Soon after the discovery of the neutron [94], Fermi introduced a pointlike $n$-nuclear $(n A)$ pseudopotential [95] for description of coherent scattering of slow neutrons: $U(\vec{r})=\left[\left(2 \pi \hbar^{2}\right) / m\right] b_{n A} \delta(\vec{r})$, with $m$ the reduced neutron mass and $b_{n A}$ the complex scattering length. Then the interaction of $n$ with matter is described using formal perturbation theory with complex optical (Fermi) potential $U(r)=\left[\left(2 \pi \hbar^{2}\right) / m\right](\rho / \mu) b_{n A}$, with $\rho$ the mass density of material and $\mu$ the atomic mass. The potential $U(r)$ for composite materials is the weighted sum of potentials from the different nuclei. It is known that a small grazing angle reflection of $n$ from materials with positive potential allows the construction of $n$ guides [96]. This applies also to $\bar{n}$ reflections with only difference that $\operatorname{Im}[U(r)]$ values are important due to $\bar{n}$ annihilation. The important parameters for the analysis of $n-\bar{n}$ oscillation experiments are the probabilities of $n$ and $\bar{n}$ reflection per bounce $\left(\rho_{n}, \rho_{\bar{n}}\right)$ and the relative phase shift between the $n$ and $\bar{n}$ wave functions per bounce $\Delta \varphi_{n \bar{n}}=\varphi_{n}-\varphi_{\bar{n}}$, where $\varphi_{n}$ and $\varphi_{\bar{n}}$ are phase shifts of the wave function upon reflection from the bulk for $n$ and $\bar{n}$. The parameters $\rho_{n}, \rho_{\bar{n}}, \varphi_{n}, \varphi_{\bar{n}}$ depend on the energy of $n / \bar{n}$ transverse motion in vacuum (e) and on the potentials of the wall material $U_{n}=V_{n}+i W_{n}, U_{\bar{n}}=$ $V_{\bar{n}}+i W_{\bar{n}}$ for the $n$ and $\bar{n}$, where $V_{n}$ and $V_{\bar{n}}$ are real parts, $W_{n}$ and $W_{\bar{n}}$ are the imaginary parts. The reflection probabilities $\rho_{n}, \rho_{\bar{n}}$ are

$$
\begin{gathered}
\rho_{n}=1, \quad \rho_{\bar{n}}=1-\frac{4 k k_{\bar{n}}^{\prime \prime}}{\left(k+k_{\bar{n}}^{\prime \prime}\right)^{2}+\left(k_{\bar{n}}^{\prime}\right)^{2}}, \\
k_{\bar{n}}^{\prime}-i k_{\bar{n}}^{\prime \prime}=\sqrt{2 m\left(V_{\bar{n}}-i W_{\bar{n}}-e\right),}
\end{gathered}
$$

$$
\begin{aligned}
& k_{\bar{n}}^{\prime}=\sqrt{m\left[\sqrt{\left(V_{\bar{n}}-e\right)^{2}+\left(W_{\bar{n}}\right)^{2}}+\left(V_{\bar{n}}-e\right)\right]}, \\
& k_{\bar{n}}^{\prime \prime}=\sqrt{m\left[\sqrt{\left(V_{\bar{n}}-e\right)^{2}+\left(W_{\bar{n}}\right)^{2}}-\left(V_{\bar{n}}-e\right)\right]},
\end{aligned}
$$

with $k_{\bar{n}}$ the complex momentum of $\bar{n}$ inside the wall. We are interested in specular reflection since its probability can reach $\sim 99.9 \%$ even for UCNs [97,98], and in $n / \bar{n}$ with transverse energies $e=\left(k^{2}\right) /(2 m)$ small compared to $V_{n}$ and $V_{\bar{n}}\left(e \ll V_{n}, e \ll V_{\bar{n}}\right)$ but comparable to $W_{\bar{n}}\left(e \sim W_{\bar{n}}\right)$. We also know from measurements of UCN reflection from highly absorbing nuclei like gadolinium that the neutron optical expression for the reflection probability works for media which possess a large imaginary component to the optical potential [99]. Based on existing theoretical analysis of the $\bar{n} A$ interaction, we know that $W_{n} \ll V_{n}, W_{\bar{n}} \ll V_{\bar{n}}$ and $W_{n} \ll W_{\bar{n}}$. It is remarkable that the reflection probability $\rho_{\bar{n}}$ (3) for the cases of weak $\left(\left|V_{\bar{n}}\right| \gg\left|W_{\bar{n}}\right|\right)$, intermediate $\left(\left|V_{\bar{n}}\right| \sim\left|W_{\bar{n}}\right|\right)$, and strong $\left(\left|V_{\bar{n}}\right| \ll\left|W_{\bar{n}}\right|\right)$ absorptions are all close to unity and quite insensitive to the variation in the magnitude of $U_{\bar{n}}$. This is consistent with known facts for analogous systems where strong losses do not destroy quantum coherence, for example in the reflection of polarized light from a metal mirror. Using these conditions, we simplify Eq. (3): $k_{\bar{n}}^{\prime} \approx \sqrt{2 m V_{\bar{n}}}$, $k_{\bar{n}}^{\prime \prime} \approx \sqrt{m\left[W_{\bar{n}}^{2} /\left(2 V_{\bar{n}}\right)\right]}$, and obtain approximate expressions for $1-\rho_{\bar{n}}$ and $\Delta \varphi_{n \bar{n}}$ :

$$
\begin{aligned}
1-\rho_{\bar{n}} & \approx \frac{4 k k_{\bar{n}}^{\prime \prime}}{\left(k_{\bar{n}}^{\prime}\right)^{2}} \\
\Delta \varphi_{n \bar{n}} & \approx \frac{2 k}{k_{n} k_{\bar{n}}^{\prime}}\left(k_{n}-k_{\bar{n}}^{\prime}\right), \\
\Delta \varphi_{n} & =\arctan \left(-\frac{2 k k_{n}}{k^{2}-k_{n}^{2}}\right), \\
\Delta \varphi_{\bar{n}} & =\arctan \left(-\frac{2 k k_{\bar{n}}^{\prime}}{k^{2}-\left(k_{\bar{n}}^{\prime}\right)^{2}-\left(k_{\bar{n}}^{\prime \prime}\right)^{2}}\right) .
\end{aligned}
$$

The low energy $\bar{n} A$ scattering is described by a scattering length $b_{\bar{n} A}$. Where direct experimental data on $\bar{n}$ were not available, the similarity between the theoretical description of $\bar{p}$ and $\bar{n}$ low-energy scattering on nuclei allowed us to adapt the fitting formula for $b_{\bar{p} A}$, derived by the authors of Ref. [76] from $\bar{p}$ experimental data to $\bar{n}$

$$
b_{\bar{p}(\bar{n}) A}=\left(1.54 A^{1 / 3}-i 1.0\right) \mathrm{fm},
$$

where $A$ the mass number. The $\sim 1$ fm size of $\operatorname{Im}\left(b_{\bar{p}(\bar{n}) A}\right)$ comes from the diffusive tail of the $\bar{n} A$ potential and is similar for all nuclei [100]. $\operatorname{Re}\left(b_{\bar{p}(\bar{n}) A}\right)$ is proportional to the nuclei radius; the $\bar{n} A$ interaction is restricted to the nuclear surface and is therefore insensitive to nuclear structure [76,100]. There is no $\bar{n}$ counterpart to compound $n A$ 
TABLE I. Parameters that characterize the interaction of $\bar{n}$ with different materials: $b_{\bar{n} A}$ (the scattering length), $U_{\bar{n}}$ (the complex optical potential for this material), $\tau_{\bar{n}}$ (the time of storage of $\bar{n}$ with close-to-zero vertical energy on a horizontal surface in Earth's gravitational field). Calculations for all elements are averaged over the natural isotopic compositions.

\begin{tabular}{llll}
\hline \hline Element & $b_{\bar{n} A}[\mathrm{fm}]$ & $U_{\bar{n}}[\mathrm{neV}]$ & $\tau_{\bar{n}}[\mathrm{~s}]$ \\
\hline $\mathrm{C}$ & $3.5-i$ & $103-i 29$ & 1.7 \\
$\mathrm{Mg}$ & $3.5-i$ & $39-i 11$ & 1.0 \\
$\mathrm{Si}$ & $3.7-i$ & $48-i 13$ & 1.2 \\
$\mathrm{Ni}$ & $4.7-i$ & $111-i 24$ & 2.3 \\
$\mathrm{Cu}$ & $4.7-i$ & $104-i 22$ & 2.2 \\
$\mathrm{Zr}$ & $5.3-i$ & $59-i 11$ & 1.8 \\
$\mathrm{Mo}$ & $5.3-i$ & $89-i 16$ & 2.3 \\
$\mathrm{~W}$ & $6.5-i$ & $106-i 16$ & 3.0 \\
$\mathrm{~Pb}$ & $6.7-i$ & $57-i 8.6$ & 2.3 \\
$\mathrm{Bi}$ & $6.7-i$ & $49-i 7$ & 2.1 \\
\hline \hline
\end{tabular}

resonances, which complicate the first-principles calculation of $b_{n A}$. The values of scattering lengths, $U_{\bar{n}}$ potentials and lifetimes of $\bar{n}$ on a surface of corresponding materials are presented in Table 1 [101]. Note that Eq. (6) is even a good approximation for mean $b_{n A}$ values as noticed in Ref. [103] and used in Ref. [104] for constraining exotic short-range forces. In fact, once the effects of resonance tails are removed, one can develop a very simple model for $n-A$ scattering amplitudes for almost all heavy nuclei that fits the measured scattering lengths to about $1 \%$ accuracy [105]. Therefore Eq. (6) is well justified in this context. The regular dependence of $b_{\bar{n} A}$ enables one to match the real parts of $U_{n}$ and $U_{\bar{n}}$. The isotopic composition ${ }^{184} W(87.7 \%)+{ }^{186}$ $W(12.3 \%)$ results in $U_{n} \sim U_{\bar{n}} \sim 106 \mathrm{neV}$.

Consider a ballistic $n$ guide [93] consisting of two parts. Its cross-sectional area $s=h d\left(\sim 10^{2} \mathrm{~cm}^{2}\right)$ at the upstream section increases along its length; let $h$ be its height and $d$ its width at the entrance, $H$ its height and $D$ its width at the exit, and $l$ the length. In the downstream part, the crosssectional area $S=H D\left(\sim 10^{4} \mathrm{~cm}^{2}\right)$ is constant over its length $L$. Since $n$ which strike the wall in the extending part see the wall recede in their rest frame, these collisions lower the transverse components of the $n$ velocity. We assume $\left|v_{\text {hor }}\right|,\left|v_{\text {vert }}\right|<2 v_{\text {crit }}^{\mathrm{Ni}}$ at the entrance, with $v_{\text {crit }}^{\mathrm{Ni}} \sim 7 \mathrm{~m} / \mathrm{s}$. In accordance with Liouville's theorem, $\quad\left|v_{\text {hor }}\right|\left|v_{\text {vert }}\right|<$ $\left(2 v_{\text {crit }}\right)^{2}(d h / D H)$ at the exit of an adiabatic guide. Values of $U_{\bar{n}}$ in Table 1 reach $>100 \mathrm{neV}$ corresponding to critical velocities of $>4 \mathrm{~m} / \mathrm{s}$. To be in the low-energy limit, we assume $\overline{\left|v_{\text {hor }}\right|} \sim \overline{\left|v_{\text {vert }}\right|} \sim 1 \mathrm{~m} / \mathrm{s}$. These conditions are met if the guide cross-sectional area is expanded by $>(D H / d h) \sim 49$. For practical arrangements, one would use a few superimposed flat guides and design a guide shape that mixes horizontal and vertical velocities of the neutrons. To account for this option, we reduce $D$ to $1 \mathrm{~m}$. Note that the diverging part contributes to the $n-\bar{n}$ sensitivity provided the $n / \bar{n}$ incidence angles are small.
We select $\mathrm{Cu}$ as a material for this analysis because $\operatorname{Re}\left(b_{\bar{n} C u}\right)$ is large, $\operatorname{Im}\left(b_{\bar{n} C u}\right)$ is relatively small, and $\mathrm{Cu}$ has been used for $n$ mirrors. $W$ with adjusted isotopic composition provides even longer storage time of $\bar{n}$.

We consider the interactions of $n / \bar{n}$ with horizontal and vertical walls separately. If $n / \bar{n}$ never touch the top, the frequencies of $n / \bar{n}$ collisions with horizontal walls and bottom are $f_{\text {hor }}=\left|v_{\text {hor }}\right| / D, f_{\text {vert }}=g /\left(2\left|v_{\text {vert }}\right|\right)$. The $\bar{n}$ lifetimes associated with sidewalls and bottom are $\tau_{\text {hor }}^{\rho, \bar{n}}=1 /\left[f_{\text {hor }}\left(1-\rho_{\bar{n}}\right)\right], \quad \tau_{\text {vert }}^{\rho, \bar{n}}=1 /\left[f_{\text {vert }}\left(1-\rho_{\bar{n}}\right)\right] ; \quad \rho_{\bar{n}}$ is given by Eq. (3). Lifetimes to reach a 1 rad phase shift between $n$ and $\bar{n}$ amplitudes associated with sidewall and bottom collisions are $\tau_{\text {hor }}^{\Delta \varphi, \bar{n}}=1 /\left(f_{\text {hor }} \Delta \varphi_{n \bar{n}}\right)$, $\tau_{\text {vert }}^{\Delta \varphi, \bar{n}}=1 /\left(f_{\text {vert }} \Delta \varphi_{n \bar{n}}\right) ; \Delta \varphi_{n \bar{n}}$ is given by Eq. (5). Thus the accumulation time for a phase difference $\Delta \varphi_{n \bar{n}}$ of $1 \mathrm{rad}$ due to the sidewall collisions, $\tau_{\text {hor }}^{\Delta \varphi, \bar{n}}$, is $32 \mathrm{~s}$ for $\mathrm{Cu}$ and very long for isotopically adjusted ${ }^{184+186} W$. The accumulation time for a phase difference $\Delta \varphi_{n \bar{n}}$ of 1 rad due to bottom collisions,

$$
\tau_{\text {vert }}^{\Delta \varphi, \bar{n}}=\frac{\overline{\left|v_{\text {vert }}\right|}}{g} \frac{\sqrt{V_{n} V_{\bar{n}}}}{\sqrt{\overline{e_{\text {vert }}}}\left|\sqrt{V_{n}}-\sqrt{V_{\bar{n}}}\right|},
$$

is $7.3 \mathrm{~s}$ for $\mathrm{Cu}$ and much longer for ${ }^{184+186} \mathrm{~W}$. For $\mathrm{Cu}$, $\tau_{\text {vert }}^{\Delta \varphi, \bar{n}} \ll \tau_{\text {hor }}^{\Delta \varphi, \bar{n}}$ because of gravity. A proper mixture of materials or isotopes for the $n / \bar{n}$ guide walls (as for $\left.{ }^{184+186} W\right)$ would increase $\tau_{\text {vert }}^{\Delta \varphi, \bar{n}}$ due to the term $\left[\left(\sqrt{V_{n}}-\right.\right.$ $\left.\sqrt{V_{\bar{n}}}\right) \rightarrow 0$ ] in the denominator of Eq. (7). The annihilation time due to the sidewall collisions, $\tau_{\text {hor }}^{\rho, \bar{n}}$, is $11 \mathrm{~s}$ for $\mathrm{Cu}$ and $15 \mathrm{~s}$ for ${ }^{184+186} \mathrm{~W}$. These timescales are large enough to neglect corresponding processes as sources of incoherence. The annihilation time due to the bottom collisions,

$$
\tau_{\mathrm{vert}}^{\rho, \bar{n}}=\frac{\overline{\left|v_{\mathrm{vert}}\right|}}{g} \frac{\left(V_{\bar{n}}\right)^{3 / 2}}{W_{\bar{n}} \sqrt{\overline{e_{\mathrm{vert}}}}},
$$

is $2.2 \mathrm{~s}$ for $\mathrm{Cu}$ and $3.1 \mathrm{~s}$ for ${ }^{184+186} W$. Annihilation of $\bar{n}$ in the accumulated reflections from the bottom is the limiting factor due to gravity. The effect of gravity is less important for a parabolic $n / \bar{n}$ guide. A smaller $g_{\text {eff }}$ value replaces $g$ in Eq. (8), and $\tau_{\text {vert }}^{\rho, \bar{n}}$ is optimized if the guide follows the trajectory of a $n / \bar{n}$ of mean velocity. The neglect of energy corrections is justified by the weak dependence of $\tau_{\text {vert }}^{\rho, \bar{n}}$ on $e_{\text {vert }}$ as the larger $n / \bar{n}$ bounce frequency and smaller probability of $\bar{n}$ annihilation per bounce nearly compensate. Therefore, the $n-\bar{n}$ experiment sensitivity is defined by one value [Eq. (8)] specific for each $n-\bar{n}$ guide material. We can estimate the impact of uncertainties in $b_{\bar{n} A}$ on the experiment sensitivity. As $\tau_{\text {vert }}^{\rho, \bar{n}} \sim 1 / W_{\bar{n}}$ [Eq. (8)], a typical $10 \%-20 \%$ error in the estimation of $W_{\bar{n}}$ gives only a $10 \%-$ $20 \%$ error in the calculation of $\tau_{\text {vert }}^{\rho, \bar{n}}$. The impact is smaller for shorter observation time. The impact of uncertainty in the $V_{\bar{n}}$ is negligible.

The above arguments treat $n / \bar{n}$ motion semiclassically. The results coincide with quantum expressions $[106,107]$ 
in the low-energy limit. The scattering length of a cold $n / \bar{n}$ is $a=1 / k_{n, \bar{n}}=1 / \sqrt{2 m\left(V_{n, \bar{n}}+W_{n, \bar{n}}\right)}$ [107]. The effective horizontal momentum of a $n / \bar{n}$ in a box with a size $D$ is $k \approx \pi j /\left(D-2 / k_{n, \bar{n}}\right)$, where $j$ is quantum number of the boxlike state. The horizonal energy levels shift is $\Delta E_{\text {hor }} \approx 4 \varepsilon_{\text {hor }} a / D=4 \varepsilon_{\text {hor }} /\left(D k_{n, \bar{n}}\right)$. The vertical energy levels shift is $\Delta E_{\mathrm{vert}}=m g a=m g / k_{n, \bar{n}}$ [107]. This expression is energy independent and consistent with the arguments given above. The quantum expressions for the timescales $\quad \tau_{\text {hor }}^{\Delta \varphi, \bar{n}}=1 / \omega_{\text {hor }}, \quad \tau_{\text {hor }}^{\rho, \bar{n}}=1 / \Gamma_{\text {a,hor }}, \quad \tau_{\text {vert }}^{\Delta \varphi, \bar{n}}=$ $1 / \omega_{\text {vert }}, \tau_{\text {vert }}^{\rho, \bar{n}}=1 / \Gamma_{\mathrm{a}, \text { vert }}$ coincide with the semiclassical expressions obtained above.

This analysis was initiated by the observation that while $\tau_{\text {vert }}^{\rho, \bar{n}}$ is short for $e_{n / \bar{n}}$ close to $V_{n / \bar{n}}$ [102], it is longer for smaller energies. However, the observation time in the limit of gravitational bound state formation [108] does not improve as the annihilation time saturates.

The total number of neutrons at PF1 beam was $3 \times 10^{18}$ in the previous free $n$ experiment [89]. The number of $n$ per year at PF1B beam is $4-5$ times larger. A gain factor due to the increased path is $\sim 10^{2}$ for $t \sim 1 \mathrm{~s}$, and $\sim 10^{4}$ for $t \sim 10 \mathrm{~s}$. Any project has to optimize the sensitivity relative to the $n / \bar{n}$ beam geometry, neutron spectrum, the budget and spatial constraints; such considerations are beyond the scope of this Letter. Assuming that detection of a couple of annihilation events in a background-free experiment means the observation of $n / \bar{n}$ oscillations, the overall sensitivity is

$$
\tau_{n \rightarrow \bar{n}} \sim \frac{\sqrt{F T}}{\Gamma_{a}},
$$

with $F$ the total $n$ flux, and $T$ the experiment duration. For the same $F$, one prefers a softer $n$ spectrum to decrease the experiment length and take full advantage of this operating mode.

The gravitational effects on wall reflections are no longer relevant for a vertical $n / \bar{n}$ guide. Consider an upwardsdirected fountain of very cold neutrons (VCNs). Gravity increases the observation time, which includes both the rise and the fall. From the estimations above we can neglect annihilation and phase shifts in the guide walls and reach practically pure quasifree limit conditions. For a largesurface VCN source based on fluorinated nanodiamond reflectors [109,110] and a typical VCN velocity of $\sim 50 \mathrm{~m} / \mathrm{s}$, the fountain height is $\sim 125 \mathrm{~m}$ and the observation time is $\sim 10 \mathrm{~s}$. For an estimated flux density of $\sim 10^{7}-10^{8} \mathrm{VCN} / \mathrm{cm}^{2} / \mathrm{s}$, we get a competitive sensitivity and largely decrease the experiment size and cost.

Finally, we emphasize that recent and future progress in our understanding of the $\bar{n} A$ optical potential and particularly in quantifying the uncertainties of real and imaginary parts of the optical potential could significantly improve the sensitivity of the $n-\bar{n}$ experiment and lower its cost through a better choice of material for the $n / \bar{n}$ guide and also through more precise optimization of these expensive experiments. Both theoretical and experimental efforts in the understanding of $\bar{n}$ annihilation by nuclei are highly encouraged.

The work of V.V.N. and W. M. S. was supported in part by a grant from the Gordon and Betty Moore Foundation. The authors are grateful to the participants of the INT-17-69W Workshop "Neutron-antineutron oscillations: Appearance, disappearance and baryogenesis" in Seattle, WA, USA, as well as to our colleagues from GRANIT Collaboration. V. G. is grateful for support of the U.S. Department of Energy, Office of Science, Office of Nuclear Physics program under Award No. DESC0015882. W. M.S. acknowledges support from NSF PHY-1614545 and from the Indiana University Center for Spacetime Symmetries.

*Corresponding author. nesvizhevsky@ill.eu

[1] E. C. G. Stueckelberg, Helv. Phys. Acta 11, 312 (1938).

[2] A. D. Sakharov, JETP Lett. 5, 24 (1967).

[3] V. A. Kuzmin, JETP Lett. 13, 335 (1970).

[4] H. Georgi and S. L. Glashow, Phys. Rev. Lett. 32, 438 (1974).

[5] Ya. B. Zeldovich, Phys. Lett. 59A, 254 (1976).

[6] G.t' Hooft, Phys. Rev. D 14, 3432 (1976).

[7] R. N. Mohapatra and R. E. Marshak, Phys. Rev. Lett. 44, 1316 (1980).

[8] M. V. Kazarnovskii, V. A. Kuzmin, K. G. Chetyrkin, and M. E. Shaposhnikov, JETP Lett. 32, 82 (1980).

[9] T. K. Kuo and S. T. Love, Phys. Rev. Lett. 45, 93 (1980).

[10] L. N. Chang and N.-P. Chang, Phys. Lett. 92B, 103 (1980).

[11] R. N. Mohapatra and R. E. Marshak, Phys. Lett. 94B, 183 (1980).

[12] K. G. Chetyrkin, M. V. Kazarnovsky, V. A. Kuzmin, and M. E. Shaposhnikov, Phys. Lett. 99B, 358 (1981).

[13] A. D. Dolgov and Ya. B. Zeldovich, Rev. Mod. Phys. 53, 1 (1981).

[14] R. Cowsik and S. Nussinov, Phys. Lett. 101B, 237 (1981).

[15] S. Rao and R. Shrock, Phys. Lett. B 116, 238 (1982).

[16] S. P. Misra and U. Sarkar, Phys. Rev. D 28, 249 (1983).

[17] S. Rao and R. E. Shrock, Nucl. Phys. B232, 143 (1984).

[18] V. A. Kuzmin, V. A. Rubakov, and M. E. Shaposhnikov, Phys. Lett. 155B, 36 (1985).

[19] M. Fukugita and T. Yanagida, Phys. Lett. B 174, 45 (1986).

[20] M. E. Shaposhnikov, Nucl. Phys. B287, 757 (1987).

[21] A. D. Dolgov, Phys. Rep. 222, 309 (1992).

[22] S. J. Huber and Q. Shafi, Phys. Lett. B 512, 365 (2001).

[23] K. S. Babu and R. N. Mohapatra, Phys. Lett. B 518, 269 (2001).

[24] S. Nussinov and R. Schrock, Phys. Rev. Lett. 88, 171601 (2002).

[25] B. Dutta, Y. Mimura, and R. N. Mohapatra, Phys. Rev. Lett. 96, 061801 (2006).

[26] Z. Berezhiani and L. Bento, Phys. Rev. Lett. 96, 081801 (2006). 
[27] C. Bambi, A. D. Dolgov, and K. Freese, Nucl. Phys. B763, 91 (2007).

[28] K. S. Babu, P. S. Bhupal Dev, and R. N. Mohapatra, Phys. Rev. D 79, 015017 (2009).

[29] R. N. Mohapatra, J. Phys. G 36, 104006 (2009).

[30] A. D. Dolgov, Phys. At. Nucl. 73, 558 (2010).

[31] P.-H. Gu, Phys. Lett. B 705, 170 (2011).

[32] D. E. Morrissey and M. J. Ramsey-Musolf, New J. Phys. 14, 125003 (2012).

[33] J. M. Arnold, B. Fornal, and M. B. Wise, Phys. Rev. D 87, 075004 (2013).

[34] K. S. Babu, P. S. Bhupal Dev, E. C. F. S. Fortes, and R. N. Mohapatra, Phys. Rev. D 87, 115019 (2013).

[35] L. Canetti, M. Drewes, T. Frossard, and M. Shaposhnikov, Phys. Rev. D 87, 093006 (2013).

[36] A. de Gouvea, J. Herrero-Garcia, and A. Kobach, Phys. Rev. D 90, 016011 (2014).

[37] Z. Berezhiani, Eur. Phys. J. C 76, 705 (2016).

[38] Z. Berezhiani and A. Vainshtein, Phys. Lett. B 788, 58 (2019).

[39] C. Grojean, B. Shakya, J. D. Wells, and Z. Zhang, Phys. Rev. Lett. 121, 171801 (2018).

[40] J. Gustafson et al., Phys. Rev. D 91, 072009 (2015).

[41] B. Aharmim et al., Phys. Rev. D 96, 092005 (2017).

[42] HyperKamiokande Proto-Collaboration, arXiv:1805.04163.

[43] Dune Collaboration, arXiv:1307.7335.

[44] E. S. Golubeva, J. I. Barrow, and C. G. Ladd, Phys. Rev. D 99, 035002 (2019).

[45] K. S. Babu and R. N. Mohapatra, Phys. Rev. D 91, 096009 (2015).

[46] V. A. Kostelesky and N. Russell, Rev. Mod. Phys. 83, 11 (2011).

[47] K. S. Babu and R. N. Mohapatra, Phys. Rev. D 94, 054034 (2016).

[48] Yu. Kamyshkov, Eur. Phys. J. C 77, 301 (2017).

[49] S. Gardner and X. Yan, Phys. Lett. B 790, 421 (2019).

[50] Z. Berezhiani and A. Vainshtein, Phys. Lett. B 788, 58 (2019).

[51] S. Gardner and E. Jafari, Phys. Rev. D 91, 096010 (2015).

[52] S. Gardner and X. Yan, Phys. Rev. D 93, 096008 (2016).

[53] K. Fujikawa and A. Tureanu, Phys. Rev. D 94, 115009 (2016).

[54] D. McKeen and A. E. Nelson, Phys. Rev. D 94, 076002 (2016).

[55] S. Gardner and X. Yan, Phys. Rev. D 97, 056008 (2018).

[56] Z. Berezhiani, Int. J. Mod. Phys. A 19, 3775 (2004).

[57] Z. Berezhiani and L. Bento, Phys. Rev. Lett. 96, 081801 (2006).

[58] A. P. Serebrov et al., Phys. Lett. B 663, 181 (2008).

[59] A. P. Serebrov et al., Nucl. Instrum. Methods Phys. Res., Sect. A 611, 137 (2009).

[60] I. Altarev et al., Phys. Rev. D 80, 032003 (2009).

[61] Z. Berezhiani and F. Nesti, Eur. Phys. J. C 72, 1974 (2012).

[62] V. I. Lushchikov, Y. N. Pokotilo, A. V. Strelkov, and F. L. Shapiro, JETP Lett. 9, 23 (1969).

[63] A. Steyerl, Phys. Lett. B 29B, 33 (1969).

[64] Z. Berezhiani, M. Frost, Y. Kamyshkov, B. Rybolt, and L. Varriano, Phys. Rev. D 96, 035039 (2017).

[65] E. D. Davis and A. R. Young, Phys. Rev. D 95, 036004 (2017).
[66] T. Bitter et al., Nucl. Instrum. Methods Phys. Res., Sect. A 309, 521 (1991).

[67] U. Kinkel, Phys. C 54, 573 (1994).

[68] C. B. Dover, A. Gal, and J. M. Richard, Phys. Rev. D 27, 1090 (1983).

[69] C. B. Dover, A. Gal, and J. M. Richard, Nucl. Instrum. Methods Phys. Res., Sect. A 284, 13 (1989).

[70] A. Gal, Phys. Rev. C 61, 028201 (2000).

[71] J. Chung et al., Phys. Rev. D 66, 032004 (2002).

[72] E. Friedman and A. Gal, Phys. Rev. D 78, 016002 (2008).

[73] K. Abe et al., Phys. Rev. D 91, 072006 (2015).

[74] R. Golub and H. Yoshiki, Nucl. Phys. A501, 869 (1989).

[75] H. Yoshiki and R. Golub, Nucl. Phys. A536, 648 (1992).

[76] B. O. Batty, E. Friedman, and A. Gal, Nucl. Phys. A689, 721 (2001).

[77] M. L. Goldberger and K. M. Watson, Collision Theory (Wiley, New York, 1964).

[78] V. F. Sears, Phys. Rep. 82, 1 (1982).

[79] V. F. Sears, Phys. Rev. A 32, 2524 (1985).

[80] V. F. Sears, Phys. Rep. 141, 281 (1986).

[81] L. Lanz and B. Vacchini, Phys. Rev. A 56, 4826 (1997).

[82] A. Cimmino, G. I. Opat, A. G. Klein, H. Kaiser, S. A. Werner, M. Arif, and R. Clothier, Phys. Rev. Lett. 63, 380 (1989).

[83] L. Alekseev, E. G. Lapin, E. K. Leushkin, V. L. Rumyantsev, O. I. Sumbaev, and V. V. Fedorov, JETP 67, 1727 (1988).

[84] V. L. Alekseev, V. V. Fedorov, E. G. Lapin, E. K. Leushkin, V. L. Rumiantsev, O. I. Sumbaev, and V. V. Voronin, Nucl. Instrum. Methods Phys. Res., Sect. A 284, 181 (1989); V. L. Alekseev et al., JETP 69, 1083 (1989).

[85] Y. D. Han and In Gyu Koh, Phys. Lett. A 167, 341 (1992).

[86] V. V. Voronin, E. G. Lapin, S. Yu. Semenikhin, and V. V. Fedorov, JETP Lett. 74, 251 (2001).

[87] W. M. Snow et al., Phys. Rev. C 83, 022501(R) (2011).

[88] B. O. Kerbikov, Phys. At. Nucl. 66, 2178 (2003).

[89] M. Baldo-Ceolin et al., Zeit. Phys. C 63, 409 (1994).

[90] G. Fidecaro et al., Phys. Lett. 156B, 122 (1985).

[91] G. Bressi et al., Nouvo Cem. 5, 731 (1990).

[92] D. G. Phillips et al., Phys. Rep. 612, 1 (2016).

[93] H. Abele et al., Nucl. Instrum. Methods Phys. Res., Sect. A 562, 407 (2006).

[94] J. Chadwick, Nature (London) 129, 312 (1932).

[95] E. Fermi, La Ric. Sient. 7, 13 (1936).

[96] H. Maier-Leibnitz, J. Nucl. En. 17, 217 (1963).

[97] V. V. Nesvizhevsky, Nucl. Instrum. Methods Phys. Res., Sect. A 557, 576 (2006).

[98] V. V. Nesvizhevsky et al., Nucl. Instrum. Methods Phys. Res., Sect. A 578, 435 (2007).

[99] V. I. Morozov, M. I. Novopoltsev, Y. N. Panin, Y. N. Pokotilovskii, and E. V. Rogov, JETP Lett. 46, 377 (1987).

[100] V. A. Karmanov, K. V. Protasov, and A. Yu. Voronin, Eur. Phys. J. A 8, 429 (2000).

[101] Note that the value of the $\bar{n} C u$ optical potential published in Refs. [88,102] is inconsistent with the corresponding scattering length, published in the same paper, and is probably wrong.

[102] B. O. Kerbikov, A. E. Kudryavtsev, and V. A. Lensky, JETP 98, 417 (2004). 
[103] M. Peshkin and G. R. Ringo, Am. J. Phys. 39, 324 (1971).

[104] V. V. Nesvizhevsky, G. Pignol, and K. V. Protasov, Phys. Rev. D 77, 034020 (2008).

[105] S. F. Mughabghab, Atlas of Neutron Resonances, 5th ed. (Elsevier, New York, 2006), Vol. 20.

[106] A. Yu. Voronin, H. Abele, S. Baessler, V. V. Nesvizhevsky, A. K. Petukhov, K. V. Protasov, and A. Westphal, Phys. Rev. D 73, 044029 (2006).
[107] A. Yu. Voronin, P. Froelich, and V. V. Nesvizhevsky, Phys. Rev. A 83, 032903 (2011).

[108] V. V. Nesvizhevsky et al., Nature (London) 415, 297 (2002).

[109] V. V. Nesvizhevsky, U. Köster, M. Dubois, N. Batisse, L. Frezet, A. Bosak, L. Gines, and O. Williams, Carbon 130, 799 (2018).

[110] V. V. Nesvizhevsky, M. Dubois, P. Gutfreund, E. V. Lychagin, A. Y. Nezvanov, and K. N. Zhernenkov, Phys. Rev. A 97, 023629 (2018). 